ITFA-2007-42

KCL-MTH-07-13

\title{
The abelian cosets of the Heisenberg group
}

\author{
Giuseppe D'Appollonio $^{a, b}$ and Thomas Quella ${ }^{c}$ \\ a Department of Mathematics, King's College \\ The Strand, London WC2R 2LS, United Kingdom \\ ${ }^{b}$ Dipartimento di Fisica dell'Università di Cagliari, INFN Sezione di Cagliari \\ Cittadella Universitaria 09042 Monserrato, Italy \\ ${ }^{c}$ Institute for Theoretical Physics, University of Amsterdam \\ Valckenierstraat 65, 1018 XE Amsterdam, The Netherlands
}

\begin{abstract}
In this paper we study the abelian cosets of the $H_{4}$ WZW model. They coincide or are related to several interesting three-dimensional backgrounds such as the Melvin model, the conical point-particle space-times and the null orbifold. We perform a detailed CFT analysis of all the models and compute the coset characters as well as some typical three-point couplings of coset primaries.
\end{abstract}




\section{Contents}

1. Introduction 1

2. The maximally symmetric plane wave 3

3. The abelian cosets of the Heisenberg group

3.1 The Melvin and the conical space-times

3.2 The gravitational wave and the null orbifold

4. The coset characters 10

4.1 The operator content of the first class of models

4.2 The operator content of the second class of models 13

5. Interactions

6. Conclusions 19

A. The group $H_{4}$ and its representations 20

\section{Introduction}

One of the principal aims of quantum gravity is the study and possible resolution of spacetime singularities. String theory is a very prominent candidate for a theory of quantum gravity and it provides several tools to deal with quantum corrections. However even in the context of string theory the analysis of singular or time-dependent backgrounds is still a very subtle and difficult problem, both from a conceptual and a technical point of view. One of the best established approaches is to investigate the property of backgrounds whose non-linear $\sigma$-models are exact conformal field theories, since they represent exact solutions to the string equations of motion to all orders in the string tension $\alpha^{\prime}$.

A first class of models is represented by the Lorentzian orbifolds of flat space [1]. Despite their apparent simplicity, the dynamics of these models and the nature of their singularities is still poorly understood [2, 3, , , 5, 5, 6. A second class of models is provided by the gravitational waves [7]. In this case the presence of a null isometry allows to prove that the $\sigma$-model is conformally invariant and to study some of its features in the lightcone gauge, as done for instance in [8]. Sometimes it is also possible to identify the chiral algebra of the underlying CFT and to solve the model in a covariant way. The first example of this type was discovered by Nappi and Witten [9] who showed that the WZW model based on the Heisenberg group $H_{4}$ coincides with the $\sigma$-model of the maximally symmetric gravitational wave in four dimension. The exact solution of this model was given in [10, 11]. 
The $H_{4}$ WZW model is also a particular example of a third large class of exact curved backgrounds of string theory, the WZW and coset models based on non-compact groups [12, 13, 14]. For several years the lack of a proper understanding of the representation theory of the corresponding affine algebras and the technical difficulties associated with the computation of the correlation functions of a non-compact CFT, allowed only a semiclassical analysis of the geometries of these models, based on the gauged WZW Lagrangian [15]. Two prominent examples of these kind of constructions are the two-dimensional black hole [14] and the cosmological Nappi-Witten space-time [16]. The situation changed during the past few years, when the structure of the $S L(2, \mathbb{R})$ WZW model and of the $\mathrm{H}_{3}^{+}$model were finally clarified [17, 18, 19] and the CFTs of some of their cosets were analysed in more detail [20, 21]. Although all these models can only be regarded as toy models for realistic singularities, they nevertheless provide a very convenient framework to explore the behaviour of strings in situations that although still largely unfamiliar are likely to display new and interesting physical effects.

In the present paper we initiate a systematic study of the cosets of the Heisenberg group $H_{4}$. There are several reasons to single out these models. First of all the corresponding non-linear $\sigma$-models describe the propagation of strings in interesting curved space-times. They were first considered in [22, 23, 24, 25] but at that time, as in the case of $S L(2, \mathbb{R})$ and its cosets, only a semiclassical analysis was possible. We can now exploit the exact solution of the $\mathrm{H}_{4}$ WZW model [10, 11] to provide a complete analysis of the underlying CFTs and in particular to determine the operator content and the couplings of all the models. Moreover the Heisenberg group is non-compact and non-semisimple. Consequently, the structure of its cosets is remarkably rich and is worth investigating in order to gain a better understanding of the representation theory of this kind of chiral algebra. This is particularly evident in the case of the diagonal cosets [24, 26] but also for the simpler abelian cosets discussed in this paper.

The abelian cosets of the Heisenberg group provide a unifying framework for the study of several time-dependent or singular three-dimensional space-times. They fall into two main classes corresponding to two inequivalent $u(1)$ subalgebras. The models in the first class [23] have either Lorentzian or Euclidean signature. In the first case they are T-dual to the conical space-time generated by a point source in three dimensions [27] while in the second case they coincide with the Melvin model [28]. The models in the second class 222 have a null isometry and describe a gravitational wave with an infinite sequence of singularities. It is interesting to note that although these two classes of models arise from inequivalent gaugings of $H_{4}$, they are related by a singular limit that is the Penrose limit leading from the point-particle space-time to the gravitational wave.

We also show that the second class of cosets, when the spatial direction transverse to the wave is compactified on a circle, reduces in a suitable limit to the null orbifold [3] and therefore provides a new framework for the study of the singularity of this model and its possible resolutions. It is worth mentioning that the fact that the three-dimensional geometries described by the abelian cosets of the Heisenberg group are T-dual to orbifolds of flat space is not surprising. In the free-field realization of the $H_{4}$ current algebra [22], the primary fields are essentially twist fields. From this point of view the affine algebra simply 
provides a very efficient tool for organising the spectrum of the model and for computing its correlation functions [10].

Exploiting the underlying chiral algebra, we determine the operator content of all these models. This provides additional evidence for the equivalence of the abelian $H_{4}$ cosets with the three-dimensional backgrounds discussed above. As an example we show in detail how the partition function of the Melvin model [35, 36, 37, 38] can be expressed in terms of cosets characters. Moreover we show that the abelian coset that describes the three-dimensional wave is an ordinary CFT. Hence the logarithmic CFT that in 39] was conjectured to arise from a contraction of the $S U(2)$ parafermions does not seem to be directly related to this geometric background.

Finally the knowledge of the three and four-point amplitudes of the $H_{4}$ model 10 makes in principle also the dynamics of these backgrounds accessible to a detailed investigation, at least at tree level in the string coupling $g_{s}$. We explain how to evaluate a generic amplitude for the cosets and then compute explicitly some three-point couplings, leaving a more complete discussion and the analysis of four-point amplitudes to future work.

The plan of the paper is as follows. In Section 2 we introduce our conventions and review the geometry of the Nappi-Witten gravitational wave. In Section 3 we classify the possible $U(1)$ subgroups of $H_{4}$ and discuss the resulting abelian cosets emphasizing their relations with the Melvin model, the conical space-times and the null orbifold. In Section 1 we determine the coset characters and the operator content of our models. In Section 5 we explain how to evaluate the correlation functions and present the calculation of some three-point couplings. An Appendix summarizes our conventions and useful information regarding the representation theory of the affine Heisenberg algebra.

\section{The maximally symmetric plane wave}

The WZW model based on the Heisenberg group $H_{4}$ describes the propagation of a string in a homogeneous Lorentzian space which is, as first noticed in [9], a gravitational wave supported by a non-trivial flux $H_{\mu \nu \rho}$ of the two-form field $B_{\mu \nu}$. Gravitational wave metrics are usually presented in Brinkman or Rosen coordinates and in our case we have

$$
\begin{array}{ll}
d s_{b}^{2}=2 d u d v_{b}-\frac{\rho^{2}}{4} d u^{2}+d \rho^{2}+\rho^{2} d \chi^{2}, & H_{u \rho \chi}=-\rho, \\
d s_{r}^{2}=2 d u d v_{r}+\sin ^{2}(u / 2)\left(d x_{1}^{2}+d x_{2}^{2}\right), & H_{u x_{1} x_{2}}=-\sin ^{2} \frac{u}{2} .
\end{array}
$$

For the analysis of the coset models two additional coordinate systems will be particularly useful

$$
\begin{aligned}
& d s_{1}^{2}=2 d u d v_{1}-\left(a_{1} d a_{2}-a_{2} d a_{1}\right) d u+d a_{1}^{2}+d a_{2}^{2}, \quad H_{u a_{1} a_{2}}=1, \\
& d s_{2}^{2}=2 d u d v_{2}+d x^{2}+d y^{2}+2 \cos u d x d y, \quad H_{u x y}=\sin u \text {. }
\end{aligned}
$$


One can pass from one coordinate system to the other using the transformations

$$
\begin{array}{lll}
a_{1}=x+y \cos u, & a_{2}=y \sin u, & v_{1}=v_{2}+\frac{x y}{2} \sin u, \\
\zeta=e^{i u / 2}\left(a_{1}-i a_{2}\right), & v_{b}=v_{1}, \\
\zeta=x e^{i u / 2}+y e^{-i u / 2}, & v_{b}=v_{2}+\frac{x y}{2} \sin u, \\
\zeta=\left(x_{1}+i x_{2}\right) \sin u / 2, & v_{b}=v_{r}-\frac{x_{1}^{2}+x_{2}^{2}}{8} \sin u,
\end{array}
$$

where $\zeta=\rho e^{i \chi}$. The action of the non-linear $\sigma$-model associated with this gravitational wave coincides with the action of the WZW model of the $H_{4}$ group. The previous four coordinate systems correspond to the following four parametrizations of the group elements

$$
\begin{array}{ll}
g_{1}=e^{a_{1} P_{1}+a_{2} P_{2}} e^{u J+v_{1} K}, & g_{2}=e^{x P_{1}} e^{u J} e^{y P_{1}+v_{2} K}, \\
g_{b}=e^{\frac{u}{2} J} e^{\frac{\bar{\zeta}}{2} P^{-}+\frac{\zeta}{2} P^{+}} e^{\frac{u}{2} J+v_{b} K}, & g_{r}=e^{\frac{x_{2} P_{1}+x_{1} P_{2}}{2}} e^{u J} e^{-\frac{x_{2} P_{1}+x_{1} P_{2}}{2}+v_{r} K} .
\end{array}
$$

Here the anti-hermitean operators $P_{1}, P_{2}, J$ and $K$ are the four generators of the Lie algebra of the Heisenberg group and satisfy the commutation relations

$$
\left[P_{1}, P_{2}\right]=K, \quad\left[J, P_{1}\right]=P_{2}, \quad\left[J, P_{2}\right]=-P_{1} .
$$

Further details on $H_{4}$ and its representations are collected in appendix A.

The $\mathrm{H}_{4}$ WZW model was completely solved in [10, 11], where the three and fourpoint correlation functions were computed for bulk and boundary vertex operators with maximally symmetric boundary conditions. In this paper we perform a similar analysis for the abelian cosets of $H_{4}$ [22, 23]. Before studying the underlying coset chiral algebras in detail, we discuss their geometric interpretation which relies on the Lagrangian formulation of the gauged WZW models [15].

\section{The abelian cosets of the Heisenberg group}

To construct an abelian coset of the maximally symmetric plane wave, we have first to single out a specific direction in the Lie algebra of $H_{4}$. This direction, by the non-compactness

of the group manifold, can be either time-like, space-like or null. Moreover the subgroups for the left and right action of $U(1)$ on $H_{4}$ can be chosen almost independently. Let us consider two embeddings $\epsilon_{L, R}$ of a $u(1)$ algebra with generator $Q$ into the $H_{4}$ Lie algebra. If the two embeddings satisfy the constraint

$$
\left\langle\epsilon_{L}(Q) \epsilon_{L}(Q)\right\rangle=\left\langle\epsilon_{R}(Q) \epsilon_{R}(Q)\right\rangle,
$$

where the brackets denote a fixed invariant bilinear form on the $H_{4}$ Lie algebra, the gauge transformation

$$
g \mapsto e^{s \epsilon_{L}(Q)} g e^{-s \epsilon_{R}(Q)},
$$

is anomaly free and leads to a consistent gauged WZW model [15]. From the form of the gauge transformation it is clear that the coset model only depends on the conjugacy 
classes of $\epsilon_{L}(Q)$ and of $\epsilon_{R}(Q)$, since even independent left and right inner automorphisms simply result in a reparametrization of the group element. Consequently there are two main classes of abelian cosets of the $\mathrm{H}_{4}$ WZW model.

The first class consists of all the models for which both $\epsilon_{L}(Q)$ and $\epsilon_{R}(Q)$ contain the generator $J$. In this case the $u(1)$ subalgebra can be brought to a standard form by conjugation and it is generated by a linear combination of $J$ and $K$

$$
\epsilon_{L}(Q)=\alpha J+\beta K, \quad \epsilon_{R}(Q)=\bar{\alpha} J+\bar{\beta} K .
$$

The embeddings are specified by four real parameters subject to the relation $\alpha \beta=\bar{\alpha} \bar{\beta}$, so that the gauging is anomaly free. Without loss of generality we can choose $2 \alpha \beta=\xi$ where $\xi= \pm 1$, with the two values of $\xi$ corresponding to different choices for the signature of the embedded $U(1)$. As we will see in the next subsection, the coset models with $\xi=1$ coincide with the conical space-times associated with a particle in three dimensions while the coset models with $\xi=-1$ lead to the Melvin model. When one of the parameter in Eq. (3.3) vanishes, the embedded $U(1)$ is null. The corresponding coset models are extremely simple since they are flat and two-dimensional, although their algebraic construction presents a few subtleties [31]. The Lagrangian description of the models with $\alpha=\bar{\alpha}$ and $\alpha=-\bar{\alpha}$, which correspond respectively to the vector and axial gauging of the $u(1)$ current, was first considered in 23]. Let us note that the vector and the axial gauging are not related by an inner automorphism.

The second class consists of all the models for which neither $\epsilon_{L}(Q)$ nor $\epsilon_{R}(Q)$ contain the generator $J$. Without loss of generality we can choose

$$
\epsilon_{L}(Q)=P_{1}, \quad \epsilon_{R}(Q)=-P_{1} .
$$

In this case the axial and vector gauging are related by an inner automorphism. The geometric data of this model, first obtained in [22], describe a gravitational wave in three dimensions with a non-trivial dilaton field. The metric has a sequence of singularities close to which it reduces to the null orbifold considered in [3]. The agreement is precise if one compactifies the spatial direction transverse to the wave.

For the sake of completeness we mention a third class of models defined by the following embedding

$$
\epsilon_{L}(Q)=\alpha J+\frac{1}{2 \alpha} K, \quad \epsilon_{R}(Q)=P_{1},
$$

which leads to a flat two-dimensional plane. In the rest of this paper we will focus on the first two classes and describe in detail the $\alpha J+\beta K$ cosets and the $P_{1}$ cosets.

\subsection{The Melvin and the conical space-times}

Let $g \in H_{4}$ and consider the following gauge transformation

$$
g \mapsto e^{s(\alpha J+\beta K)} g e^{-s(\bar{\alpha} J+\bar{\beta} K)} .
$$

In the coordinate system (2.3) we have

$$
(u, v, r, \varphi) \mapsto(u+s(\alpha-\bar{\alpha}), v+s(\beta-\bar{\beta}), r, \varphi+s \alpha),
$$


where $a_{1}+i a_{2}=r e^{i \varphi}$. There are two convenient gauge choices. If $\alpha \neq \bar{\alpha}$ we can fix completely the gauge freedom setting $u=0$. Alternatively we can first set $\varphi=0$ and then take into account the residual discrete identifications

$$
(u, v) \sim\left(u+2 \pi k \frac{\alpha-\bar{\alpha}}{\alpha}, v+2 \pi k \frac{\beta-\bar{\beta}}{\alpha}\right), \quad k \in \mathbb{Z} .
$$

It is convenient to define the parameter $\eta$

$$
\eta=\frac{\alpha-\bar{\alpha}}{\alpha \bar{\alpha}}
$$

where we are assuming that both $\alpha$ and $\bar{\alpha}$ are non-zero. The metric, dilaton and two-form fields of the model with $\xi=1$ are

$$
d s^{2}=d r^{2}+\frac{\eta^{2} r^{2}}{\eta^{2}-r^{2}} d \varphi^{2}-\frac{4}{\eta^{2}-r^{2}} d v^{2}, \quad B=\frac{2 \eta^{2}}{\eta^{2}-r^{2}} d \varphi \wedge d v, \quad \Phi=\ln \left(\eta^{2}-r^{2}\right) .
$$

This is a three-dimensional Lorentzian space with a naked singularity and closed time-like curves in the region $r^{2}>\eta^{2}$ where the time direction is the periodic coordinate $\varphi$. The model with $\xi=-1$ corresponds to the following smooth Euclidean space

$$
d s^{2}=d r^{2}+\frac{\eta^{2} r^{2}}{\eta^{2}+r^{2}} d \varphi^{2}+\frac{4}{\eta^{2}+r^{2}} d v^{2}, \quad B=\frac{2 \eta^{2}}{\eta^{2}+r^{2}} d \varphi \wedge d v, \quad \Phi=\ln \left(\eta^{2}+r^{2}\right) .
$$

When $\alpha=\bar{\alpha}$ the geometric data of the coset are

$$
d s^{2}=-\xi d x^{2}+d r^{2}+\frac{4 d v^{2}}{r^{2}}, \quad \Phi=\ln \left(r^{2}\right),
$$

with $x=u / \alpha$. This metric can also be derived from Eq. (3.10) and Eq. (3.11) setting $x=\eta \varphi$ and taking the limit $\eta \rightarrow 0$.

The space-times in Eq. (3.10) and Eq. (3.11) are T-dual to freely acting orbifolds of flat space. The model with $\xi=-1$ is in fact easily recognized as the Melvin model [28, 33, 34, 35, 36]. Let us start with a flat three-dimensional Euclidean space

$$
d s^{2}=d x^{2}+d r^{2}+r^{2} d \theta^{2}
$$

and identify the points related by the transformation $(x, \theta) \mapsto(x+2 \pi R, \theta+2 \pi \gamma)$ with $R \in \mathbb{R}$ and $\gamma \in[0,1)$. We then define a new coordinate $\varphi=\theta-\frac{\gamma}{R} x$ and perform a T-duality along the $x$ direction. The resulting background coincides with (3.11) if we set $\eta=\frac{R}{\gamma}$ and $2 v \gamma=R \tilde{x}$, where $\tilde{x}$ is the coordinate T-dual to $x$. As such the coordinate $v$ has radius $R_{v}=\frac{1}{\gamma}$ so that the generic Melvin background coincides with an abelian coset of the $H_{4}$ model with a compact $v$ direction. The existence of this relation between the $H_{4}$ model and the Melvin space-time was first noticed in [36].

The model with $\xi=1$ is T-dual to the conical space-time generated by a point mass in three dimensions [27]. This is equivalent to a three-dimensional Minkowski space $d s^{2}=$ $-d t^{2}+d r^{2}+r^{2} d \theta^{2}$ where the points related by the transformation $(t, \theta) \mapsto(t-2 \pi R, \theta+2 \pi \gamma)$ are identified. The mass and angular momentum of the source are

$$
m=\frac{1-\gamma}{4 \pi G_{N}}, \quad L=\frac{R}{4 G_{N}},
$$


where $G_{N}$ is the Newton constant. In order to compare these conical space-times and the models in Eq. (3.11) we follow exactly the same steps as before, introducing a new coordinate $\varphi=\theta+\frac{\gamma}{R} t$ and then performing a T-duality along the $t$ direction. The relations between the parameters of the two models are $\eta=\frac{R}{\gamma}, \eta \tilde{t}=2 v$ and $v \sim v-2 \pi / \gamma$, so that the background generated by a point particle with generic mass and angular momentum coincides with an abelian coset of the $H_{4}$ model with a compact $v$ direction of radius $R_{v}=1 / \gamma$.

Also the model in Eq. (3.12) is T-dual to a flat three-dimensional space. To see this one first writes the metric of the two-plane in polar coordinates and then perfoms a T-duality along the angular direction. This model was discussed in [40] as a toy model to study T-duality transformations in non-compact spaces.

As a final observation, let us stress that the abelian cosets of $H_{4}$ can be related to suitable limits of the two-dimensional charged black-hole and the three dimensional black string [42, 43, 44] or more generally to abelian cosets of models given by the product of $U(1), S U(2)$ and $S L(2, \mathbb{R})$ WZW models. This is due to the fact that the affine algebra based on the Heisenberg group is a contraction of the product of $\widehat{S U}(2)$ and a time-like free boson or of $\widehat{S L}(2, \mathbb{R})$ and a space-like free boson [30].

\subsection{The gravitational wave and the null orbifold}

Let us consider now the second class of abelian cosets and gauge the $u(1)$ subalgebra generated by $P_{1}$. Let $g \in H_{4}$ and consider the gauge transformation

$$
g \mapsto e^{s P_{1}} g e^{\mp s P_{1}} .
$$

The two choices of sign correspond respectively to the vector and axial gauging of the $U(1)$ current and are related by an inner automorphism. In the coordinate system (2.4) the transformation is simply $x \mapsto x+s, y \mapsto y \mp s$, so that we can fix the gauge freedom setting $x=y$ for the vector model and $x=-y$ for the axial model. The background fields of the cosets are easily obtained and describe a three-dimensional gravitational wave supported by a dilaton [22]. For the axial gauging we have

$$
d s^{2}=2 d u d v+4 \tan ^{2} \frac{u}{2} d x^{2}, \quad \Phi=\ln \cos ^{2} \frac{u}{2},
$$

where the result is automatically expressed in Rosen coordinates. The coordinate transformation

$$
v=V+\frac{X^{2}}{2 \sin u}, \quad x=\frac{X}{2} \cot \frac{u}{2},
$$

brings the metric into Brinkman form

$$
d s^{2}=2 d u d V+\frac{X^{2}}{2 \cos ^{2} \frac{u}{2}} d u^{2}+d X^{2}, \quad \Phi=\ln \cos ^{2} \frac{u}{2} .
$$

The background fields for the vector gauging are related to the previous ones by $u \rightarrow \pi-u$, $v \rightarrow-v$. The metric is singular at the points $u_{n}=n \pi$. The $R_{u x u x}$ component of the Riemann tensor of the axial model diverges at the points $u_{2 n+1}$ where the model is also 
strongly coupled. Around these points a better description of the background geometry is provided by the T-dual vector model.

We show now that in a neighbourhood of $u=0$ the metric and the wave functions of the $P_{1}$ cosets reduce to the corresponding quantities of the null orbifold considered in [3]. In order to take the limit in a controlled way we first rescale the coordinates

$$
(u, v, x) \rightarrow(\zeta u, v / \zeta, x / \zeta),
$$

and then send the parameter $\zeta$ to zero. In this limit the dilaton of the axial model tends to a constant while the metric becomes

$$
d s^{2}=2 d u d v+u^{2} d x^{2}, \quad d s^{2}=2 d u d V+d X^{2} .
$$

The null orbifold is a Lorentzian orbifold of flat space, a class of models introduced in [1]. It owes its name to the fact that the orbifold group is generated by a null Lorentz transformation, namely a linear combination of a boost and a rotation. We start from $d s^{2}=2 d u d V+d X^{2}$ and identify the points related by

$$
(X, V) \sim\left(X+2 \pi \nu u, V-2 \pi \nu X-2 \pi^{2} \nu^{2} u\right),
$$

where the value of the parameter $\nu$ can be set to one by a boost. The change of coordinates

$$
X=u x, \quad V=v-\frac{u}{2} x^{2},
$$

brings the metric into the form (3.20) with the identification $x \sim x+2 \pi \nu$. Therefore in order to have precise agreement with the geometry of the $P_{1}$ coset we must compactify the coordinate $x$ of the latter on a circle of radius $R$. If we denote by $i \lambda$ and $i \bar{\lambda}$ the eigenvalues of $P_{1}$, the axial model contains only winding states with $\lambda+\bar{\lambda}=0$ while the vector model contains only momentum states with $\lambda-\bar{\lambda}=0$. A discrete spectrum containing both winding and momentum modes requires a compact $x$ direction. In this case the operators that survive in the spectrum have

$$
\lambda=\frac{n}{R}+\frac{w R}{2}, \quad \bar{\lambda}=\frac{n}{R}-\frac{w R}{2} .
$$

It is this compactified coset model with radius $R=1 / \zeta$ that precisely matches the null orbifold in the limit $\zeta \rightarrow 0$. Note that unlike the parameter $\nu$ of the null orbifold, the radius $R$ labels different models and cannot be set to one by a boost.

To illustrate this correspondence further let us show that the wave functions of the states of the coset become in the limit the semiclassical wave functions of the null orbifold. The wave function of a state in $H_{4}$ with $K=i p, P_{1}=i \lambda, \bar{P}_{1}=i \bar{\lambda}$ and Casimir $C$ is

$$
\psi_{p, C, \lambda, \bar{\lambda}}=\frac{1}{\sqrt{|\sin u|}} e^{-i p v-i \frac{C}{p} u-i \lambda x+i \bar{\lambda} y-\frac{i}{4 p}\left[(\lambda+\bar{\lambda})^{2} \cot \frac{u}{2}-(\lambda-\bar{\lambda})^{2} \tan \frac{u}{2}\right]} .
$$

In order to reduce this wave functions to the coset we set $y=-x$. In Brinkman coordinates the result is

$$
\psi_{p, C, \lambda, \bar{\lambda}}=\frac{1}{\sqrt{|\sin u|}} e^{-i p V-i \frac{C}{p} u-i \frac{p}{4} \tan \frac{u}{2}\left[X^{2}-\frac{(\lambda-\bar{\lambda})^{2}}{p^{2}}\right]-i \frac{p}{4} \cot \frac{u}{2}\left[X^{2}+\frac{\lambda+\bar{\lambda}}{p}\right]^{2}} .
$$


We now rescale the coordinates and take the limit $\zeta \rightarrow 0$ keeping $\tilde{p}=p / \zeta$ finite in the limit. Up to a shift in the value of the Casimir $C$ the wave function becomes

$$
\psi_{\tilde{p}, C, n, w} \sim \frac{1}{\sqrt{|u|}} e^{-i \tilde{p} V-i \frac{C}{\tilde{p}} u-i \frac{\tilde{p}}{2 u}\left(X+\frac{2 n}{p}\right)^{2}+\frac{i}{4 \tilde{p}} \frac{w^{2}}{24} u^{3}} .
$$

Following the notation of [3], the previous expression coincides with the wave function of a state of the null orbifold with winding number $w$, momentum $J=n$ and light-cone momentum $p^{+}=\tilde{p} / 2$. Moreover, $u=-2 x^{+}$and $V=x^{-} / 2$. All the states whose wave function is given by Eq. (3.24) have $p \neq 0$. The wave functions of states with $p=0$ are distributions localized at the points $u_{n}$ and can be identified with the states that live on the singular plane crossing the tip of the two cones of the null orbifold.

It is interesting to note that this background is also the Penrose limit of the point particle space-times, that is of the $\alpha J+\beta K$ cosets with $\xi=1$. If we set

$$
r=\eta \sin \frac{u}{2}, \quad \varphi=\frac{x}{\eta}, \quad v=\frac{\eta^{2}}{8}(u+\sin u)-t
$$

the new coordinates parametrize the region $r<\eta$. In the limit $\eta \rightarrow \infty$ we magnify the neighbourhood of a null geodesic and the space-time reduces to the three-dimensional gravitational wave discussed in this section. Although the $U(1)$ subgroup generated by $P_{1}$ and the one generated by $\alpha J+\beta K$ with $\xi=1$ are not equivalent the relation

$$
\alpha J+\frac{1}{2 \alpha} K=e^{-\frac{P_{2}}{\alpha}}\left(\alpha J+P_{1}\right) e^{\frac{P_{2}}{\alpha}},
$$

implies that they are connected by the singular limit $\alpha \rightarrow 0$. This limit is the algebraic equivalent of the Penrose limit and it is very similar to the relation between the elliptic orbifold $\mathbb{R} \times \mathbb{C} / \mathbb{Z}_{N}$ and the null orbifold discussed in [3, 6].

As a final remark let us mention that this gravitational wave can also be obtained as the Penrose limit of the parafermions times a time-like free boson. The background fields of the coset $S U(2)_{k} / U(1)_{k} \times \mathbb{R}$ are

$$
d s^{2}=-k d t^{2}+k\left[d \theta^{2}+\tan ^{2} \theta d \varphi^{2}\right], \quad \Phi=\ln \cos ^{2} \theta,
$$

and if we set

$$
u=t+\theta, \quad \frac{2 v}{k}=-t+\theta, \quad \frac{2 x}{\sqrt{k}}=\varphi,
$$

and consider the limit $k \rightarrow \infty$ we obtain (3.16). From the point of view of the theory on the world-sheet, the Penrose limit is a contraction of the chiral algebra. The contractions of $S U(2)_{k} \times U(1)$ to $H_{4}$ and of $S L(2, \mathbb{R})_{k} \times S U(2)_{k}$ to $H_{6}$ were studied in detail in 10,29 . It would be interesting to study also the $P_{1}$ coset as a contraction of the chiral algebra of the parafermions and a time-like boson. This point of view was adopted in [39] where the authors defined a contracted chiral algebra starting with a superposition of parafermionic and $u(1)$ operators whose conformal dimensions agree only in the limit. When one expands the OPEs in a power series in the contraction parameter, the subleading difference in the conformal weights yields additional terms that are typical for a logarithmic CFT. This 
observation led the authors of [39] to conjecture that the resulting theory is a logarithmic CFT with $c=3$.

It seems worthwhile to point out that the limit so defined cannot correspond to the geometric background described in this paper. In fact, the abelian $H_{4}$ coset is certainly not a logarithmic CFT since the dilatation operator $L_{0}$ is completely diagonalisable on the full state space. This can most easily be understood on the level of the minisuperspace theory where $L_{0}$ reduces to the Laplacian acting on a suitable space of functions. For the coset this space coincides with the space of functions on the Heisenberg group which are invariant under the action of the subgroup. Also, the coset Laplacian agrees with that of the Heisenberg group up to a contribution proportional to $P_{1}^{2}$. Both of these operators are completely diagonalisable, thus proving our assertion. Presumably this property persists in the full CFT and the correlation functions of the primary vertex operators satisfy the standard conformal Ward identities. A non-diagonalisable term in $L_{0}$ could appear if one considers the supersymmetric version of this coset or even the supersymmetric $H_{4}$ WZW model itself. This is due to the fact that the world-sheet fermions transform in the adjoint representations of the Heisenberg group which is not fully decomposable. A similar nondiagonalisable term in $L_{0}$ was discussed in the context of the null orbifold of the RNS superstring [6].

\section{The coset characters}

The knowledge of the exact CFT underlying the curved backgrounds discussed in the previous section allows us to compute the spectrum of the vertex operators and their correlation functions. When these CFTs are used to build a consistent string background, we can determine both the exact spectrum of perturbative string excitations and their interactions. The operator content of the abelian cosets of $H_{4}$ can be derived from the spectrum of the $H_{4}$ model [22, 32, 10]. The spectrum of a coset conformal field theory $G / H$ is given by a collection of usually irreducible representations of the coset chiral algebra. Any representation $\mu$ of the affine Lie algebra $\hat{\mathfrak{g}}$ associated to $G$ can in fact be decomposed into representations of the affine subalgebra $\hat{\mathfrak{h}} \subset \hat{\mathfrak{g}}$ belonging to the subgroup $H$. The resulting branching spaces give rise to representations of the coset chiral algebra, the commutant of $\hat{\mathfrak{h}}$ in the universal enveloping algebra $\mathcal{U}(\hat{\mathfrak{g}})$. In the following we shall describe the decomposition of the $\hat{H}_{4}$ representations with respect to the two abelian subalgebras that have been used to construct the backgrounds discussed in the previous section. For a review of the representation theory of the affine Lie algebra $\hat{H}_{4}$ we refer the reader to Appendix A.

\subsection{The operator content of the first class of models}

The characters of the first class of models can be obtained from the $\hat{H}_{4}$ characters in a straightforward way. This is due to the fact that $J$ and $K$ are the generators of the standard Cartan subalgebra of $H_{4}$. The character formulas for the coset representations resemble closely those appearing in the context of the two-dimensional black hole [45, 46]. We will decompose the $\hat{H}_{4}$ characters with respect to the $\hat{u}(1)$-subalgebra defined by the injection

$$
\epsilon(Q)=\alpha J+\beta K
$$


with $2 \alpha \beta=\xi \in\{ \pm 1\}$ depending on whether we are gauging a space-like or a time-like isometry. It follows from this condition that the map $\epsilon$ is an embedding of the full current algebra

$$
Q(z) Q(w)=\frac{\xi}{(z-w)^{2}}
$$

Via $\epsilon$ any $\hat{H}_{4}$-representation $\mu$ can be interpreted as a reducible $\hat{u}(1)$-representation and the irreducible constituents $\chi_{[\mu, b]}^{H_{4} / U(1)}(q)$ can be determined by writing

$$
\begin{aligned}
\operatorname{tr}_{\mu}\left[q^{L_{0}-\frac{c}{24}} z^{-i \epsilon\left(Q_{0}\right)}\right] & =\operatorname{tr}_{\mu}\left[q^{L_{0}-\frac{c}{24}} z^{-i \alpha J_{0}} z^{-i \beta K_{0}}\right]=\chi_{\mu}^{H_{4}}\left(q, z^{\alpha}, z^{\beta}\right) \\
& =\sum_{b} \chi_{[\mu, b]}^{H_{4} / U(1)}(q) \chi_{b}^{U(1)}(q, z)
\end{aligned}
$$

where in the last expression we introduced the standard $\hat{u}(1)$ characters of charge $b$

$$
\chi_{b}^{U(1)}(q, z)=\frac{q^{\frac{\xi}{2} b^{2}} z^{b}}{\eta(q)} .
$$

For the continuous series of representations, Eq. (4.3) leads immediately to the coset characters

$$
\chi_{\left[(0, \sigma, j)_{-\omega}, b_{n}^{0}\right]}^{H_{4} / U(1)}(q)=\frac{q^{h_{(0, \sigma, j)}+\omega(j+n)-\frac{\xi}{2}\left(b_{n}^{0}\right)^{2}}}{\eta(q)^{3}}, \quad \omega \in \mathbb{Z},
$$

where the charge $b_{n}^{0}$ is given by

$$
b_{n}^{0}=\alpha(j+n)+\frac{\xi}{2 \alpha} \omega .
$$

The conformal dimension of the corresponding coset representation is

$$
h_{\left[(0, \sigma, j)_{-\omega}, b_{n}^{0}\right]}^{H_{4} / U(1)}=h_{(0, \sigma, j)}+\omega(j+n)-\frac{\xi}{2}\left(b_{n}^{0}\right)^{2} .
$$

In order to obtain the characters of the discrete series we first expand $\chi_{( \pm, p, j)_{\omega}}^{H_{4}}\left(q, z^{\alpha}, z^{\beta}\right)$ according to Eq. (A.15) and then regroup the $z$-dependence. The resulting expression reads

$$
\chi_{\left[( \pm, p, j)_{\mp \omega}, b_{n}^{ \pm}\right]}^{H_{4} / U(1)}(q)=\sum_{m=0}^{\infty}(-1)^{m} \frac{q^{h_{( \pm, p, j)}+\frac{m}{2}(m+2 n+1) \pm \omega(j \mp n)-\frac{\xi}{2}\left(b_{n}^{+}\right)^{2}}}{\eta(q)^{3}},
$$

where the $U(1)$ charges $b_{n}^{ \pm}$are

$$
b_{n}^{ \pm}=\alpha(j \mp n) \pm \frac{\xi}{2 \alpha}(p+\omega)
$$

If $n \geq 0$ the minimal contribution to the $q$-series comes from $m=0$. On the contrary for $n<0$ the first few terms in the series cancel and the minimum of the exponent is reached for a positive half-integer value of $m$. Altogether we end up with the conformal dimensions

$$
h_{\left[( \pm, p, j)_{\mp \omega}, b_{n}^{ \pm}\right]}^{H_{4} / U(1)}= \begin{cases}h_{( \pm, p, j)} \pm \omega(j \mp n)-\frac{\xi}{2}\left(b_{n}^{ \pm}\right)^{2} & , n \geq 0 \\ h_{( \pm, p, j)} \pm \omega(j \mp n)-\frac{\xi}{2}\left(b_{n}^{ \pm}\right)^{2}-n & , n<0 .\end{cases}
$$


The states of the WZW model survive the coset projection precisely when $Q=\bar{Q}$. When $\alpha \neq \bar{\alpha}$ it is convenient to label the states in the decomposition of a discrete representation with the values of $p, \omega, n$ and $\bar{n}$ and then use the previous constraint to fix $j$

$$
j= \pm \xi \frac{p+\omega}{2 \alpha \bar{\alpha}} \pm \frac{\alpha n-\bar{\alpha} \bar{n}}{\alpha-\bar{\alpha}} .
$$

In a similar way we label the states in the decomposition of a continuous representation with the values of $\sigma, \omega$ and of $L=n-\bar{n}$. This set of quantum numbers identifies the state uniquely, since the values of $j \in[0,1)$ and $n+\bar{n}$ are given by the constraint

$$
j+n=\xi \frac{\omega}{2 \alpha \bar{\alpha}}-\frac{\bar{\alpha} L}{\alpha-\bar{\alpha}} .
$$

When $\alpha=\bar{\alpha}$ the constraint $Q=\bar{Q}$ reduces to $n=\bar{n}$ and therefore we can specify $p, j$, $\omega$ and $n$ for the states that appear in the decomposition of the discrete representations $( \pm, p, j)_{\mp \omega}$, and $\sigma, \omega, j$ and $n$ for the states that appear in the decomposition of the continuous representations $(0, \sigma, j)_{-\omega}$.

The spectrum of this class of coset models can be neatly summarised by the torus partition function. When $\alpha \neq \bar{\alpha}$ we obtains

$$
\begin{aligned}
\mathcal{Z}_{H_{4} / U(1)} & =\sum_{\omega=0}^{\infty} \int_{0}^{1} d p \sum_{n, \bar{n} \in \mathbb{Z}}\left(\left|\chi_{\left[(+, p, j)_{-\omega}, b_{n}^{+}\right]}^{H_{4} / U(1)}(q)\right|^{2}+\left|\chi_{\left[(-, p, j)_{\omega}, b_{n}^{-}\right]}^{H_{4} / U(1)}(q)\right|^{2}\right) \\
& +\sum_{\omega \in \mathbb{Z}}^{\infty} \int_{0}^{\infty} d s s \int_{0}^{2 \pi} d \beta \sum_{L \in \mathbb{Z}}\left|\chi_{\left[(0, \sigma, j)_{-\omega}, b_{n}^{0}\right]}^{\left.H_{4} / U\right)}\right|^{2},
\end{aligned}
$$

where the value of the quantum numbers that do not appear in the sums or in the integrals is assumed to be fixed by (4.11) or (4.12). We now discuss the case $\xi=-1$ in more detail in order to show the precise relation between the abelian $H_{4}$ coset and the Melvin model. Using Eq. (4.5) and (4.8), the contribution of the discrete representations can be written as

$$
\begin{aligned}
\mathcal{Z}_{H_{4} / U(1)}^{\text {discrete }}= & \sum_{\omega \in \mathbb{Z}} \int_{0}^{1} d p \sum_{n, \bar{n} \in \mathbb{Z}} q^{\frac{1}{2}\left[\frac{\eta}{2}(p+\omega)-\frac{n-\bar{n}}{\eta}\right]^{2}+\frac{p}{2}(1-p)+n p} \bar{q}^{\frac{1}{2}\left[\frac{\eta}{2}(p+\omega)-\frac{n-\bar{n}}{\eta}\right]^{2}+\frac{p}{2}(1-p)+\bar{n} p} \\
& \frac{1}{[\eta(\tau) \eta(\bar{\tau})]^{3}} \sum_{m, \bar{m}=0}^{\infty}(-1)^{m+\bar{m}} q^{\frac{m}{2}(m+2 n+1)} \bar{q}^{\frac{\bar{m}}{2}(\bar{m}+2 \bar{n}+1)}
\end{aligned}
$$

and the contribution of the continuous representations as

$$
\mathcal{Z}_{H_{4} / U(1)}^{\text {continuous }}=\frac{1}{[\eta(\tau) \eta(\bar{\tau})]^{3}} \sum_{\omega, L \in \mathbb{Z}} \int_{0}^{2 \pi} d \beta \int_{0}^{\infty} d s s q^{\frac{s^{2}}{2}+\frac{1}{2}\left[\frac{\eta}{2} \omega-\frac{L}{\eta}\right]^{2}} \bar{q}^{\frac{s^{2}}{2}+\frac{1}{2}\left[\frac{\eta}{2} \omega+\frac{L}{\eta}\right]^{2}} .
$$

We want to compare Eq. (4.14) with the contribution of the twisted sectors to the partition function of the Melvin model. The partition function of the Melvin model has been discussed in several papers [36, 37, 38] since it provides an interesting example of a noncompact orbifold. The contribution of the twisted sectors can be written, setting $\alpha^{\prime}=2$,

$$
\mathcal{Z}_{\text {Melvin }}^{\text {twisted }}=\sum_{s, t \in \mathbb{Z}, s \neq 0} \int_{-\infty}^{\infty} \frac{d l}{2 \pi} q^{\frac{1}{2}(l+s R / 2)^{2}} \bar{q}^{\frac{1}{2}(l-s R / 2)^{2}} e^{2 \pi i(l R) t}\left|\vartheta\left[\begin{array}{c}
1 / 2+s \gamma \\
1 / 2+t \gamma
\end{array}\right](0 \mid \tau)\right|^{-2},
$$


where $\vartheta[a, b](z \mid \tau)$ is the Jacobi $\vartheta$-function. Using the identity (A.16) we can write this partition function in the following form which allows a simple comparison with the contribution of the discrete representations in the coset partition function

$$
\begin{aligned}
\mathcal{Z}_{\text {Melvin }}^{\text {twisted }}= & \sum_{s, k \in \mathbb{Z}, s \neq 0} \sum_{n, \bar{n} \in \mathbb{Z}} q^{\frac{1}{2}\left[\frac{\gamma(n-\bar{n})+k}{R}-\frac{s R}{2}\right]^{2}+\frac{\{\gamma s\}}{2}(1-\{\gamma s\})} \bar{q}^{\frac{1}{2}\left[\frac{\gamma(n-\bar{n})+k}{R}+\frac{s R}{2}\right]^{2}+\frac{\{\gamma s\}}{2}(1-\{\gamma s\})} \\
& \frac{q^{n\{\gamma s\}} \bar{q}^{\bar{n}\{\gamma s\}}}{[\eta(\tau) \eta(\bar{\tau})]^{3}} \sum_{m, \bar{m}=0}^{\infty}(-1)^{m+\bar{m}} q^{\frac{m}{2}(m+2 n+1)} \bar{q}^{\frac{\bar{m}}{2}(\bar{m}+2 \bar{n}+1)}
\end{aligned}
$$

where $\{a\}$ stands for the fractional part of the real number $a$. As explained in the previous section the $H_{4} / U(1)$ model corresponds to the limit

$$
\frac{R}{\gamma} \rightarrow \eta, \quad \gamma \rightarrow 0
$$

of the Melvin model. In this small radius limit the momentum modes labeled by $k$ decouple and the discrete sum over the twisted sectors becomes an integral in a continuous variable that can be identified with $p+\omega$.

When $\gamma$ is an irrational number, the states in a twisted sector with $\gamma s$ very close to an integer are almost delocalized. The limit of these almost delocalized twisted states are the spectral flowed continuous representations of the coset model.

In order to reproduce the partition function of the Melvin model with a finite radius $R=\eta \gamma$, we have to compactify the $v$ direction of the $H_{4}$ model on a circle of radius

$R_{v}=1 / \gamma$. As a result the quantum numbers $p+\omega$ and $\zeta \equiv j-\bar{j}$ can only be multiples of the compactification radius and its inverse

$$
p+\omega=s \gamma, \quad \zeta=\frac{k}{\gamma},
$$

and the partition function

$$
\begin{aligned}
\mathcal{Z}_{H_{4} / U(1)}^{\text {discrete }}= & \sum_{s, k \in \mathbb{Z}} \sum_{n, \bar{n} \in \mathbb{Z}} q^{\frac{1}{2}\left[\frac{\eta}{2}(p+\omega)-\frac{n-\bar{n}+\zeta}{\eta}\right]^{2}+\frac{p}{2}(1-p)+n p} \bar{q}^{\frac{1}{2}\left[\frac{\eta}{2}(p+\omega)-\frac{n-\bar{n}-\zeta}{\eta}\right]^{2}+\frac{p}{2}(1-p)+\bar{n} p} \\
& \frac{1}{[\eta(\tau) \eta(\bar{\tau})]^{3}} \sum_{m, \bar{m}=0}^{\infty}(-1)^{m+\bar{m}} q^{\frac{m}{2}(m+2 n+1)} \bar{q}^{\frac{\bar{m}}{2}(\bar{m}+2 \bar{n}+1)}
\end{aligned}
$$

agrees precisely with (4.17).

The partition function of the model with $\alpha=\bar{\alpha}$ and of the conical space-times can be discussed along similar lines.

\subsection{The operator content of the second class of models}

The irreducible representations of the Heisenberg group are classified by the values of the central element $K$ and of the Casimir operator $C$. Until now we have labeled the states in a given representation using a discrete label $n$ related to their $J$ eigenvalues. More precisely the states in the representation $( \pm, p, j)$ have $J$-eigenvalues $j \mp n, n \in \mathbb{N}$ and 
are denoted by $| \pm, p, j ; n\rangle$ while the states in the representation $(0, \sigma, j)$ have $J$-eigenvalues $j+m, m \in \mathbb{Z}$ and are denoted by $|0, \sigma, j ; m\rangle$. Instead of diagonalising the generator $J$, we can also diagonalise $P_{1}$. If we do so, the states in each irreducible representation carry a continuous label $\lambda$ since the spectrum of the generator $P_{1}$ is continuous. To study the characters of the $P_{1}$ coset it is natural to choose this continuous basis. The relation between the basis of $J$ and $P_{1}$ eigenstates is quite simple

$$
\begin{aligned}
& | \pm, p, j ; \lambda\rangle=\frac{e^{-\frac{\lambda^{2}}{2 p}}}{(\pi p)^{1 / 4}} \sum_{n=0}^{\infty} \frac{H_{n}(\lambda / \sqrt{p})}{2^{\frac{n}{2}} \sqrt{n !}}| \pm, p, j ; n\rangle, \\
& | \pm, p, j ; n\rangle=\int_{-\infty}^{\infty} d \lambda \frac{e^{-\frac{\lambda^{2}}{2 p}}}{(\pi p)^{1 / 4}} \frac{H_{n}(\lambda / \sqrt{p})}{2^{\frac{n}{2}} \sqrt{n !}}| \pm, p, j ; \lambda\rangle,
\end{aligned}
$$

where $\lambda \in \mathbb{R}$ and the functions $H_{n}(t)$ are the Hermite polynomials. It is easy to verify that $P_{1}|\lambda, p, j\rangle=i \lambda|\lambda, p, j\rangle$. Using Mehler's formula we can evaluate the following trace on a representation of the horizontal subalgebra

$$
\operatorname{tr}_{( \pm, p, j)}\left(z^{-i J} x^{-i P_{1}}\right)=\int d \lambda x^{\lambda} \tau_{( \pm, p)}(z, \lambda) \chi_{( \pm, p, j)}(z)
$$

where

$$
\tau_{(-, p)}(z, \lambda)=\frac{1}{\sqrt{\pi p}} \sqrt{\frac{1+z}{1-z}} e^{-\frac{\lambda^{2}}{p} \frac{1-z}{1+z}},
$$

and $\tau_{(+, p)}(z, \lambda)=\tau_{(-, p)}(1 / z, \lambda)$. Similar relations hold for the continuous representations. In this case we can construct the states

$$
|0, \sigma, j ; \theta\rangle=\sum_{m \in \mathbb{Z}} e^{i m \theta}|0, \sigma, j ; m\rangle, \quad \theta \in[0,2 \pi)
$$

which are eigenstates of $P_{1}$ with eigenvalue $\lambda=s \cos (\beta+\theta)$. The evaluation of the trace gives in this case

$$
\operatorname{tr}_{(0, \sigma, j)}\left(z^{-i J} x^{-i P_{1}}\right)=\frac{1}{\pi} \int_{-s}^{s} \frac{d \lambda x^{\lambda}}{\sqrt{s^{2}-\lambda^{2}}} \sum_{n \in \mathbb{Z}} z^{j+n}
$$

In order to decompose the $\hat{H}_{4}$ characters with respect to the subalgebra generated by $P_{1}$ we first study the decomposition of the affine representations $\hat{\mu}$ with respect to the horizontal subalgebra. This is easily done using once more eq. (A.16). For the discrete representations we obtain

$$
\chi_{( \pm, p, j)_{\mp \omega}}^{H_{4}}(q, z)=\sum_{n \in \mathbb{Z}} \frac{z^{j \mp n}}{1-z^{\mp 1}} q^{h_{( \pm, p, j)} \pm \omega(j \mp n)} \sum_{m=1}^{\infty}(-1)^{m+1} \frac{q^{\frac{m}{2}(m+2 n-1)}}{\eta(q)^{4}}\left(q^{\omega}-q^{m}\right) .
$$

Once the character is written in this form, we can easily read the multiplicity of the representation $( \pm, p, j \mp n)$ of the horizontal subalgebra at each level of the affine representation. 
We then use this decomposition and the trace in (4.23) to evaluate the following modified $\hat{H}_{4}$-character

$$
\begin{gathered}
\tilde{\chi}_{( \pm, p, j)_{\mp \omega}}^{H_{4}}(q, z, x) \equiv \operatorname{tr}_{( \pm, p, j)_{\mp \omega}}\left(q^{L_{0}-\frac{c}{24}} z^{-i J_{0}} x^{-i\left(P_{1}\right)_{0}}\right)=\sum_{n \in \mathbb{Z}} \int d \lambda x^{\lambda} \tau_{( \pm, p+\omega)}(z, \lambda) \\
\chi_{( \pm, p, j \mp n)}(z) q^{h_{( \pm, p, j)} \pm \omega(j \mp n)} \sum_{m=1}^{\infty}(-1)^{m+1} \frac{q^{\frac{m}{2}(m+2 n-1)}}{\eta(q)^{4}}\left(q^{\omega}-q^{m}\right) .
\end{gathered}
$$

Finally, in order to extract the coset characters we set $z=1$ and remove the contribution of the affine $u(1)$ character. The function $\tau_{( \pm, p+\omega)}(z, \lambda)$ is divergent for $z=1$ but this divergence can be absorbed in the normalization of the states of the non-compact $U(1)$. The result is

$$
\chi_{\left[( \pm, p, j)_{\mp \omega}, \lambda\right]}^{H_{4} / U(1)}(q)=\sum_{n \in \mathbb{Z}} q^{h_{( \pm, p, j)} \pm \omega(j \mp n)-\frac{\lambda^{2}}{2}} \sum_{m=1}^{\infty}(-1)^{m+1} \frac{q^{\frac{m}{2}(m+2 n-1)}\left(q^{\omega}-q^{m}\right)}{\eta(q)^{3}} .
$$

When $\omega=0$ the character can be written in the simple form

$$
\chi_{[( \pm, p, j), \lambda]}^{H_{4} / U(1)}(q)=\frac{q^{h_{( \pm, p, j)}-\frac{\lambda^{2}}{2}}}{\eta(q)^{3}} .
$$

Following the same strategy as outlined above we obtain from the decomposition of the continuous representations the characters

$$
\chi_{\left[(0, \sigma, j)_{-\omega, \lambda]}\right.}^{H_{4} / U(1)}(q, z, u)=\sum_{n \in \mathbb{Z}} \frac{q^{h_{(0, s, j)}-\frac{\lambda^{2}}{2}+\omega(j+n)}}{\eta(q)^{3}} z^{j+n},
$$

where $\lambda$ can assume the values $\lambda=s \cos \theta$ with $0 \leq \theta<2 \pi$. The conformal dimensions of the coset representations are therefore

$$
h_{\left[( \pm, p, j)_{\mp \omega} ; \lambda\right]}= \pm(p+\omega) j+\frac{p}{2}(1-p)-\frac{\lambda^{2}}{2},
$$

with $\omega \in \mathbb{N},(\lambda, j) \in \mathbb{R}^{2}, p \in(0,1)$ and

$$
h_{\left[(0, s, j)_{-\omega ; \lambda]}\right.}=\omega j+\frac{s^{2}-\lambda^{2}}{2},
$$

with $\omega \in \mathbb{Z}, s \in \mathbb{R}_{+}, \lambda \in[-s, s], j \in[0,1)$. The spectral flowed representations, as it is evident from their characters, contain states with arbitrarily negative values of $L_{0}$.

\section{Interactions}

The correlation functions of a coset model $G / H$ can be expressed in terms of the correlation functions of the $G$ and $H$ WZW models (see for instance [47]). When applied to the vertex operators, the decomposition of the $G$ representations in a sum of products of coset and $H$ representations gives

$$
\Phi_{g}^{G}(z)=\sum_{h} \Phi_{[g, h]}^{G / H}(z) \Phi_{h}^{H}(z)
$$


where $\Phi_{g}^{G}, \Phi_{h}^{H}$ and $\Phi_{[g, h]}^{G / H}$ are respectively primary fields of the two WZW models and of the coset model. To simplify the notation we are displaying only the chiral part of the vertex operators. A general $n$-point correlator can then be written in a factorized form

$$
\left\langle\prod_{i=1}^{n} \Phi_{g_{i}}^{G}\left(z_{i}\right)\right\rangle=\sum_{h_{i}}\left\langle\prod_{i=1}^{n} \Phi_{\left[g_{i}, h_{i}\right]}^{G / H}\left(z_{i}\right)\right\rangle\left\langle\prod_{i=1}^{n} \Phi_{h_{i}}^{H}\left(z_{i}\right)\right\rangle,
$$

from which one can extract the correlators of the coset model and in particular the two and three-point couplings. Since the three and four-point correlation functions of the $H_{4}$ WZW model are known [10], we can use these results to evaluate some typical correlators for the abelian cosets discussed in the previous sections. We restrict our attention to correlators that do not involve spectral flowed states.

Let us denote a primary field of $H_{4}$ as $\Phi_{(q, n, \bar{n})}^{H_{4}}$, where $q$ stands for all the quantum numbers necessary to specify the representation of $\hat{H}_{4}$ and $n, \bar{n}$ keep track of the $J$ and $\bar{J}$ eigenvalues of the field. With this notation $(q, n, \bar{n})$ identifies the state with eigenvalues $j \mp n$ and $j \mp \bar{n}$ if $q=( \pm, p, j)$ and with eigenvalues $j+n$ and $j+\bar{n}$ if $q=(0, \sigma, j)$. The correlation functions assume a simpler form if one introduces charge variables to handle simultaneously all the fields that belong to the same representation [48]. For fields in a discrete representation we use a complex variable $x$. The monomials

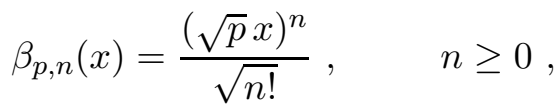

provide a complete orthonormal basis for the space of analytic functions $f(x)$ with measure

$$
d \sigma_{p}=\frac{d^{2} x}{\pi} p e^{-p x^{*} x},
$$

where $x^{*}$ is the complex conjugate of $x$. We also introduce a complex charge variable $\bar{x} \in \mathbb{C}$ for the antichiral fields. For fields in a continuous representation we consider the phases $\beta_{0, n}(\theta)=e^{i n \theta}$ with $\theta \in[0,2 \pi), n \in \mathbb{Z}$ and use the measure $d \sigma_{0}=d \theta / 2 \pi$. We can now collect all the primary fields that belong to the same representation in a single field that depends both on the worldsheet and the charge variables

$$
\Phi_{q}^{H_{4}}(z, \bar{z}, x, \bar{x})=\sum_{n, \bar{n}} \Phi_{(q, n, \bar{n})}^{H_{4}}(z, \bar{z}) \beta_{q, n}(x) \beta_{q, \bar{n}}(\bar{x}),
$$

with $n, \bar{n} \in \mathbb{N}$ for the discrete representations and $n, \bar{n} \in \mathbb{Z}$ for the continuous representations. The three-point couplings of these primary fields are given by

$$
\left\langle\prod_{i=1}^{3} \Phi_{q_{i}}^{H_{4}}\left(x_{i}, \bar{x}_{i}\right)\right\rangle=\mathcal{K}_{q_{1}, q_{2}, q_{3}}\left(x_{1}, x_{2}, x_{3}\right) \mathcal{K}_{q_{1}, q_{2}, q_{3}}\left(\bar{x}_{1}, \bar{x}_{2}, \bar{x}_{3}\right) \mathcal{C}_{q_{1}, q_{2}, q_{3}} .
$$

Here and in the following we leave understood the standard dependence on the insertion points $z_{i}$ of the vertex operators, which is completely fixed by the Ward identities of global conformal invariance. The explicit form of the functions $\mathcal{K}_{q_{1}, q_{2}, q_{3}}$ and $\mathcal{C}_{q_{1}, q_{2}, q_{3}}$ can be found in $10,29,11 .{ }^{1}$

\footnotetext{
${ }^{1}$ The $( \pm, p, j)$ representations in the conventions of 10, 29, 11] correspond to the $(\mp, p, j)$ representations in the conventions of the present paper.
} 
Let us consider first some examples of three-point couplings for the $\alpha J+\beta K$ cosets. In this case we need the couplings between the fields $\Phi_{(q, n, \bar{n})}^{H_{4}}$ that can be easily extracted from the couplings in Eq. (5.6) using the orthonormality of the functions $\beta_{q, n}$ and the quantities

$$
\mathcal{K}_{q_{1}, q_{2}, q_{3}}^{n_{1}, n_{2}, n_{3}} \equiv \prod_{i=1}^{3} \int d \sigma_{q_{i}} \beta_{q_{i}, n_{i}}^{*}\left(x_{i}\right) \mathcal{K}_{q_{1}, q_{2}, q_{3}}\left(x_{1}, x_{2}, x_{3}\right) .
$$

Consider the coupling between the coset fields $\left[\left(-, p_{1}, j_{1}\right), b_{n_{1}}^{-}, b_{\bar{n}_{1}}^{-}\right],\left[\left(-, p_{2}, j_{2}\right), b_{n_{2}}^{-}, b_{\bar{n}_{2}}^{-}\right]$and $\left[\left(+, p_{3}, j_{3}\right), b_{n_{3}}^{+}, b_{\bar{n}_{3}}^{+}\right]$, where the charges $b_{n}^{ \pm}$are as defined in Eq. (4.6) and Eq. (4.9). In the following we assume for simplicity that all the labels $n_{i}$ and $\bar{n}_{i}$ are non-negative, otherwise one needs the couplings of descendant fields of the $H_{4}$ WZW model. When the $q$ labels take the previous values, the $H_{4}$ coupling is non-zero only when $p_{3}=p_{1}+p_{2}$ and $\Delta \equiv-\left(j_{1}+j_{2}+j_{3}\right) \in \mathbb{N}$. In this case we have

$$
\mathcal{K}_{q_{1}, q_{2}, q_{3}}=e^{-x_{3}\left(p_{1} x_{1}+p_{2} x_{2}\right)}\left(x_{2}-x_{1}\right)^{\Delta}, \quad \mathcal{C}_{q_{1}, q_{2}, q_{3}}=\frac{1}{\Delta !}\left[\frac{\gamma\left(p_{3}\right)}{\gamma\left(p_{1}\right) \gamma\left(p_{2}\right)}\right]^{\Delta},
$$

where $\gamma(x)=\frac{\Gamma(x)}{\Gamma(1-x)}$. The three-point coupling of the coset fields is then given by $\left\langle\Phi_{\left[\left(-, p_{1}, j_{1}\right), b_{n_{1}}^{-}, b_{\bar{n}_{1}}^{-}\right]}^{H_{4} / U(1)} \Phi_{\left[\left(-, p_{2}, j_{2}\right), b_{n_{2}}^{-}, b_{\bar{n}_{2}}^{-}\right]}^{H_{4} / U(1)} \Phi_{\left[\left(+, p_{3}, j_{3}\right), b_{n_{3}}^{+}, b_{\bar{n}_{3}}^{+}\right]}^{H_{4} / U(1)}\right\rangle=\frac{1}{\Delta !}\left[\frac{\gamma\left(p_{1}+p_{2}\right)}{\gamma\left(p_{1}\right) \gamma\left(p_{2}\right)}\right]^{\Delta} \mathcal{K}_{q_{1}, q_{2}, q_{3}}^{n_{1}, n_{2}, n_{3}} \mathcal{K}_{q_{1}, q_{2}, q_{3}}^{\bar{n}_{1}, \bar{n}_{2}, \bar{n}_{3}}$,

where $\Delta=n_{1}+n_{2}-n_{3}=\bar{n}_{1}+\bar{n}_{2}-\bar{n}_{3}$ and

$$
\mathcal{K}_{q_{1}, q_{2}, q_{3}}^{n_{1}, n_{2}, n_{3}}=\sqrt{\frac{n_{2} !}{n_{1} ! n_{3} !}} \frac{\Delta !(-1)^{n_{1}+n_{3}}}{\left(n_{2}-n_{3}\right) !} \frac{p_{2}^{n_{3}}}{p_{1}^{n_{1} / 2} p_{2}^{n_{2} / 2} p_{3}^{n_{3} / 2}} F\left(-n_{1},-n_{3} ; n_{2}-n_{3}+1 ;-p_{1} / p_{2}\right),
$$

when $n_{2} \geq n_{3}$ and

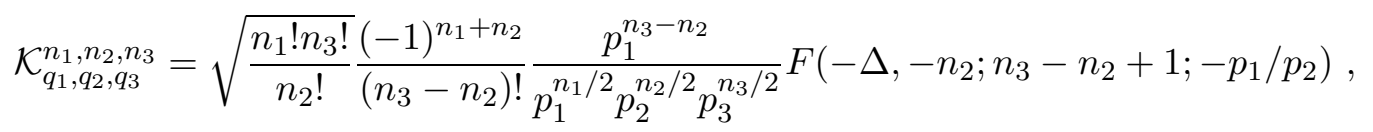

when $n_{3} \geq n_{2}$. Here $F(a, b ; c ; t)$ is the hypergeometric function. As a second example we consider the coupling between the coset fields $\left[\left(-, p_{1}, j_{1}\right), b_{n_{1}}^{-}, b_{\bar{n}_{1}}^{-}\right],\left[\left(+, p_{2}, j_{2}\right), b_{n_{2}}^{+}, b_{\bar{n}_{2}}^{+}\right]$and $\left[\left(0, \sigma, j_{3}\right), b_{n_{3}}^{0}, b_{\bar{n}_{3}}^{0}\right]$ and for simplicity we assume again that the labels $n_{i}$ and $\bar{n}_{i}, i=1,2$ are non-negative. In this case we have a non-vanishing $H_{4}$ coupling only if $p_{1}=p_{2} \equiv p$ and $\Delta \equiv-\left(j_{1}+j_{2}+j_{3}\right) \in \mathbb{Z}$. Moreover

$$
\mathcal{K}_{q_{1}, q_{2}, q_{3}}=e^{-p x_{1} x_{2}-\frac{1}{\sqrt{2}}\left(\sigma x_{2} x_{3}+\sigma^{*} \frac{x_{1}}{x_{3}}\right)} x_{3}^{\Delta}, \quad \mathcal{C}_{q_{1}, q_{2}, q_{3}}=e^{\frac{s^{2}}{2} \Sigma(p)},
$$

where

$$
\Sigma(p)=\psi(p)+\psi(1-p)-2 \psi(1), \quad \psi(x)=\frac{\mathrm{d} \ln \Gamma(x)}{d x} .
$$

When these conditions are satisfied the three-point coupling in the coset model can be written as

$$
\left\langle\Phi_{\left[\left(-, p, j_{1}\right), b_{n_{1}}^{-}, b_{\bar{n}_{1}}^{-}\right]}^{H_{4} / U(1)} \Phi_{\left[\left(+, p, j_{2}\right), b_{n_{2}}^{+}, b_{\bar{n}_{2}}^{+}\right]}^{H_{4} / U(1)} \Phi_{\left[\left(0, \sigma, j_{3}\right), b_{n_{3}}^{0}, b_{\bar{n}_{3}}^{0}\right]}^{H_{4} / U(1)}\right\rangle=e^{\frac{s^{2}}{2} \Sigma(p)} \mathcal{K}_{q_{1}, q_{2}, q_{3}}^{n_{1}, n_{2}, n_{3}} \mathcal{K}_{q_{1}, q_{2}, q_{3}}^{\bar{n}_{1}, \bar{n}_{2}, \bar{n}_{3}}
$$


where $\Delta=n_{1}-n_{2}+n_{3}=\bar{n}_{1}-\bar{n}_{2}+\bar{n}_{3}$ and

$$
\mathcal{K}_{q_{1}, q_{2}, q_{3}}^{n_{1}, n_{2}, n_{3}}=\left(\frac{s}{\sqrt{2 p}}\right)^{n_{1}+n_{2}} \sum_{k=0}^{m} \frac{(-1)^{k+n_{1}+n_{2}} \sqrt{n_{1} ! n_{2} !}}{k !\left(n_{2}-k\right) !\left(n_{1}-k\right) !}\left(\frac{2 p}{s^{2}}\right)^{k}
$$

with $m=\min \left(n_{1}, n_{2}\right)$. Following the same procedure one can compute various other couplings among coset fields.

Let us consider now the second class of models. The best way to compute the couplings of the $P_{1}$ coset is to first rewrite the $H_{4}$ couplings in a basis of $P_{1}$ and $\bar{P}_{1}$ eigenstates. To this end we apply the change of basis in Eq. (4.21) to the states

$$
| \pm, p, j, x, \bar{x}\rangle=\sum_{n, \bar{n}=0}^{\infty} \beta_{p, n}(x) \beta_{p, \bar{n}}(\bar{x})| \pm, p, j, n, \bar{n}\rangle \text {. }
$$

The result is

$$
\begin{aligned}
& | \pm, p, j, x, \bar{x}\rangle=\int_{-\infty}^{\infty} d \lambda \int_{-\infty}^{\infty} d \bar{\lambda} G_{p}(x, \lambda) G_{p}(\bar{x}, \bar{\lambda})| \pm, p, j, \lambda, \bar{\lambda}\rangle, \\
& | \pm, p, j, \lambda, \bar{\lambda}\rangle=\int_{\mathbb{C}} d \sigma_{p} \int_{\mathbb{C}} d \bar{\sigma}_{p} G_{p}^{*}(x, \lambda) G_{p}^{*}(\bar{x}, \bar{\lambda})| \pm, p, j, x, \bar{x}\rangle
\end{aligned}
$$

where

$$
G_{p}(x, \lambda)=\frac{1}{(\pi p)^{1 / 4}} e^{-\frac{\lambda^{2}}{2 p}+\sqrt{2} \lambda x-\frac{p}{2} x^{2}}, \quad \lambda \in \mathbb{R}, x \in \mathbb{C} .
$$

For the continuous representations we consider the states

$$
|0, \sigma, j, \theta, \bar{\theta}\rangle=\sum_{n, \bar{n} \in \mathbb{Z}} \beta_{0, n}(\theta) \beta_{0, \bar{n}}(\bar{\theta})|0, \sigma, j, n, \bar{n}\rangle,
$$

which are eigenstates of $P_{1}$ with eigenvalue $\lambda=s \cos (\theta+\bar{\theta}+\beta)$. Once the $H_{4}$ couplings have been expressed in a basis of $P_{1}$ eigenstates, the couplings of the coset fields can be easily obtained by removing the contribution of the $U(1)$ vertex operators and setting

$$
\lambda=\frac{n}{R}+\frac{m R}{2}, \quad \bar{\lambda}=\frac{n}{R}-\frac{m R}{2},
$$

if we consider the general case of the compact coset with $x \sim x+2 \pi R$. The condition imposed on the states by the vector gauging $(\lambda=\bar{\lambda})$ and the condition imposed on the states by the axial gauging $(\lambda=-\bar{\lambda})$ arise respectively in the limit $R \rightarrow \infty$ and $R \rightarrow 0$. This is of course what we expect, since the axial and the vector model are related by T-duality. We present here just two examples of three-point couplings. The first one is

$$
\begin{aligned}
& \left\langle\Phi_{\left[\left(-, p_{1}, j_{1}\right), \lambda_{1}, \bar{\lambda}_{1}\right]}^{H_{4} / U(1)} \Phi_{\left[\left(-, p_{2}, j_{2}\right), \lambda_{2}, \bar{\lambda}_{2}\right]}^{H_{4} / U(1)} \Phi_{\left[\left(+, p_{3}, j_{3}\right), \lambda_{3}, \bar{\lambda}_{3}\right]}^{H_{4} / U(1)}\right\rangle= \\
& \frac{1}{\Delta !}\left[\frac{\gamma\left(p_{3}\right)}{\gamma\left(p_{1}\right) \gamma\left(p_{2}\right)}\right]^{\Delta+\frac{1}{2}}\left[\frac{p_{3}}{2 p_{1} p_{2}}\right]^{\Delta} e^{-\sum_{i=1}^{3} \frac{\lambda_{i}^{2}+\bar{\lambda}_{i}^{2}}{2 p_{i}}} H_{\Delta}(\mu) H_{\Delta}(\bar{\mu}),
\end{aligned}
$$

where $p_{3}=p_{1}+p_{2}, \Delta \in \mathbb{N}, \lambda_{1}+\lambda_{2}+\lambda_{3}=\bar{\lambda}_{1}+\bar{\lambda}_{2}+\bar{\lambda}_{3}=0$ and the argument $\mu$ of the Hermite polynomial $H_{\Delta}$ is

$$
\mu \equiv \sqrt{\frac{\lambda_{1}^{2}}{p_{1}}+\frac{\lambda_{2}^{2}}{p_{2}}-\frac{\lambda_{3}^{2}}{p_{3}}}
$$


The quantity $\bar{\mu}$ has exactly the same expression in terms of the barred quantities. Our second example is

$$
\begin{aligned}
& \left\langle\Phi_{\left[\left(-, p, j_{1}\right), \lambda_{1}, \bar{\lambda}_{1}\right]}^{H_{4} / U(1)} \Phi_{\left[\left(+, p, j_{2}\right), \lambda_{2}, \bar{\lambda}_{2}\right]}^{H_{4} / U(1)} \Phi_{\left[\left(0, \sigma, j_{3}\right), \lambda_{3}, \bar{\lambda}_{3}\right]}^{H_{4} / U(1)}\right\rangle= \\
& e^{-\frac{\lambda_{1} \lambda_{2}}{2 p}-\frac{\lambda_{3}^{2}}{2 p} \tan ^{2} \theta+\frac{i \lambda_{3}\left(\lambda_{1}-l a_{2}\right)}{p} \tan \theta} e^{-\frac{\bar{\lambda}_{1} \bar{\lambda}_{2}}{2 p}-\frac{\bar{\lambda}_{3}^{2}}{2 p} \tan ^{2} \theta+\frac{i \bar{\lambda}_{3}\left(\bar{\lambda}_{1}-\bar{\lambda}_{2}\right)}{p} \tan \theta} e^{\frac{s^{2}}{2} \Sigma(p)},
\end{aligned}
$$

where $\Delta \in \mathbb{Z}, \lambda_{1}+\lambda_{2}+\lambda_{3}=\bar{\lambda}_{1}+\bar{\lambda}_{2}+\bar{\lambda}_{3}=0$ and $\lambda_{3}=s \cos (\theta+\beta), \bar{\lambda}_{3}=s \cos (\bar{\theta}+\beta)$.

\section{Conclusions}

In this article we reported on progress in understanding various space-times arising from gauging a one-dimensional subgroup of the Heisenberg group $H_{4}$. All the models we obtained are non-compact and many of them in fact exhibit time-dependence and an interesting structure of curvature singularities. Building on the solution of the $H_{4}$ WZW model [10, 11], we have been able to derive exact conformal field theory results such as spectra and correlation functions.

The specific backgrounds we discussed came in two families. In order to obtain the first class we gauged a subgroup generated by a linear combination of the currents $J$ and $K$. This resulted in models which are T-dual to freely acting orbifolds of flat space. Depending on the signature we recovered either the Melvin model [28] or the conical space-times that are generated by point particles in three-dimensional gravity [27]. For the Melvin model we showed explicitly that the coset partition function coincides with the partition functions of a freely acting orbifold of flat space [35, 36].

The second class of models was obtained by gauging the subgroup generated by $P_{1}$. Vector and axial gauging turned out to be equivalent in this case and led to a gravitational wave with a periodic array of singularities. While these two gaugings lead to a continuous spectrum of either momentum or winding modes, we also considered a compactified version of the model, with a discrete spectrum of momenta and windings. We showed, both at the level of the geometry and of the semi-classical wave functions, that this compact model reduces in a suitable limit to the null orbifold of Minkowski space [3]

Our work invites for generalizations in several directions. For a better understanding of the models and of their singularities one should study in detail their dynamics. The role played by the twisted sectors of the Lorentzian orbifolds has been the object of several papers [3, 5, 6] and led to some proposals for the fate of their singularities [6], inspired by the analogy with previous work on tachyon condensation in non-compact Euclidean orbifolds [49]. It would be interesting to study the amplitudes of the compact $P_{1}$ coset discussed in this paper and to compare them with the results obtained for the null orbifold [3]. It would also be worth investigating the space of the possible relevant and marginal deformations of these models, taking for instance as a starting point the current-current deformations of the $\mathrm{H}_{4}$ WZW model discussed in [10], since the cosets considered here are the boundary points of such deformations.

Additional insights on the physical properties of these space-times may be gained by the analysis of the dynamics of open strings. Although in the present work we restricted 
ourselves to the closed string sector, the entire analysis could be extended to cover open strings and D-branes. In fact the boundary CFTs associated with the maximally symmetric branes of the Heisenberg group were solved in [11] and therefore the corresponding spectra and correlation functions are known. Together with the formalism developed for the description of D-branes in asymmetrically gauged WZW models [50], these results should lead to a clear picture of open strings in the abelian $H_{4}$ cosets.

Finally, it remains to say that the insights and techniques gained and developed here can also be used to study more complicated, non-abelian cosets based on the Heisenberg group. This concerns in particular its diagonal cosets which have been shown to correspond to non-maximally symmetric plane waves in four dimensions [24]. We shall return to this class of models in a forthcoming publication [26].

\section{Acknowledgments}

The authors are grateful to Sylvain Ribault and Volker Schomerus for discussions. G.D. acknowledges the support of the PPARC rolling grant PP/C507145/1. The research of T.Q. is funded by a Marie Curie Intra-European Fellowship, contract number MEIF-CT2007-041765. We also acknowledge partial support from the EU Research Training Network Superstring theory, MRTN-CT-2004-512194.

\section{A. The group $H_{4}$ and its representations}

The Lie algebra of the group $H_{4}$ has four generators that satisfy the following commutation relations

$$
\left[P_{1}, P_{2}\right]=K, \quad\left[J, P_{1}\right]=P_{2}, \quad\left[J, P_{2}\right]=-P_{1},
$$

or, in terms of the raising and lowering operators $P^{ \pm}=P_{1} \pm i P_{2}$,

$$
\left[P^{+}, P^{-}\right]=-2 i K, \quad\left[J, P^{ \pm}\right]=\mp i P^{ \pm} .
$$

All generators are anti-hermitean and the invariant bilinear form is

$$
\left\langle P^{+}, P^{-}\right\rangle=-2, \quad\langle J, K\rangle=-1 .
$$

The corresponding current algebra is

$$
\begin{aligned}
P^{+}(z) P^{-}(w) & =-\frac{2}{(z-w)^{2}}-\frac{2 i K}{z-w}, \quad J(z) K(w)=-\frac{1}{(z-w)^{2}}, \\
J(z) P^{ \pm}(w) & =\mp \frac{i P^{ \pm}}{z-w},
\end{aligned}
$$

or, in terms of the modes,

$$
\left[P_{n}^{+}, P_{m}^{-}\right]=-2 n \delta_{n+m, 0}-2 i K_{n+m}, \quad\left[J_{n}, P_{m}^{ \pm}\right]=\mp i P_{n+m}^{ \pm}, \quad\left[J_{n}, K_{m}\right]=-n \delta_{n+m, 0} .
$$

The energy momentum tensor

$$
T=-\frac{1}{4}\left[P^{+} P^{-}+P^{-} P^{+}\right]-J K+\frac{K^{2}}{2},
$$


generates a Virasoro algebra with central charge $c=4$.

There are three classes of irreducible unitary representations of the group $H_{4}$ [22]: two families of so-called discrete series representations $( \pm, p, j)$ (with $p>0$ and $j \in \mathbb{R}$ ) and one family of continuous series representations $(0, \sigma, j)$ (with $\sigma=s e^{i \beta}, s \geq 0,0 \leq$ $\beta<2 \pi$ and $j \in[0,1))$. All these representations are infinite dimensional and their weight content is clearly displayed by their characters $\chi(z, u)=\operatorname{tr}\left[z^{-i J} u^{-i K}\right]$. The discrete series representations $( \pm, p, j)$ are constructed respectively from a highest and a lowest weight

$$
\begin{aligned}
& \chi_{(+, p, j)}(z, u)=\sum_{n \geq 0} z^{j-n} u^{p}=\frac{z^{j} u^{p}}{1-1 / z} \\
& \chi_{(-, p, j)}(z, u)=\sum_{n \geq 0} z^{j+n} u^{-p}=\frac{z^{j} u^{-p}}{1-z} .
\end{aligned}
$$

The Casimir operator $C=-\frac{1}{2}\left(P^{+} P^{-}+P^{-} P^{+}\right)-2 J K$ assumes the values $C=p(1 \pm 2 j)$ on these representations. The continuous series representations $(0, \sigma, j)$ possess neither a highest nor a lowest weight state and the generator $K$ acts trivially on them so that their characters are simply given by

$$
\chi_{(0, \sigma, j)}(z, u)=\sum_{n \in \mathbb{Z}} z^{j+n} .
$$

The additional label $\sigma$ gives the value of the Casimir, $C=s^{2}$.

We now review the representation theory of the affine Lie algebra $\hat{H}_{4}$. The simplest class of representations of $\hat{H}_{4}$ is generated by the action of all the negative modes on the unitary representations of the horizontal subalgebra. These Verma modules turn out to be irreducible. Let us recall that the Cartan subalgebra of $\hat{H}_{4}$ is generated by the current zero modes $J_{0}$ and $K_{0}$ and by the Virasoro generator $L_{0}$. Following our prescription for the horizontal subalgebra we thus introduce the character of a representation $\mu$ as follows,

$$
\chi_{\mu}^{\hat{H}_{4}}(q, z, u)=\operatorname{tr}_{\mu}\left[q^{L_{0}-\frac{c}{24}} z^{-i J_{0}} u^{-i K_{0}}\right] .
$$

Their explicit form is easily derived

$$
\begin{aligned}
& \chi_{(+, p, j)}^{\hat{H}_{4}}(q, z, u)=\frac{q^{h_{(+, p, j)}-\frac{1}{12} z^{j} u^{p}}}{\left(1-z^{-1}\right) \eta(q)^{2} \prod_{n=1}^{\infty}\left(1-z q^{n}\right)\left(1-z^{-1} q^{n}\right)} \quad\left(|q|^{-1}>|z|>1\right) \\
& \chi_{(-, p, j)}^{\hat{H}_{4}}(q, z, u)=\frac{q^{h_{(-, p, j)}-\frac{1}{12} z^{j} u^{-p}}}{(1-z) \eta(q)^{2} \prod_{n=1}^{\infty}\left(1-z q^{n}\right)\left(1-z^{-1} q^{n}\right)} \quad(|q|<|z|<1) \\
& \chi_{(0, \sigma, j)}^{\hat{H}_{4}}(q, z, u)=\frac{q^{h_{(0, \sigma, j)}-\frac{1}{12}} \sum_{n \in \mathbb{Z}} z^{j+n}}{\eta(q)^{2} \prod_{n=1}^{\infty}\left(1-z q^{n}\right)\left(1-z^{-1} q^{n}\right)}=\frac{q^{h_{(0, \sigma, j)}}}{\eta(q)^{4}} \sum_{n \in \mathbb{Z}} z^{j+n} .
\end{aligned}
$$

We will refer to the modules $( \pm, p, j)$ and $(0, \sigma, j)$ as standard representations. Furthermore, we restrict the range of the label $p$ of the $( \pm, p, j)$ representations to the interval $(0,1)$. 
The conformal weights of the ground states transforming in the representations $( \pm, p, j)$ and $(0, \sigma, j)$ are given by

$$
h_{( \pm, p, j)}=\frac{p}{2}(1-p) \pm p j \quad, \quad h_{(0, \sigma, j)}=\frac{s^{2}}{2} .
$$

These value agree with the eigenvalue of the "improved" Casimir operator $\frac{1}{2}\left(C+K^{2}\right)$ that appears in the quantum corrected energy momentum tensor.

In addition to the standard representations, $\hat{H}_{4}$ also admits representations which in some loose sense can be referred to as twisted highest weight representations. Their construction rests on the observation that the $\hat{H}_{4}$ current algebra (A.5) allows for a spectral flow automorphism which acts on the modes as (see [10] for instance)

$$
\Omega_{\omega}\left(P_{n}^{ \pm}\right)=P_{n \mp \omega}^{ \pm} \quad \Omega_{\omega}\left(J_{n}\right)=J_{n} \quad \Omega_{\omega}\left(K_{n}\right)=K_{n}+i \omega \delta_{n 0} .
$$

From this definition one readily derives the action

$$
\Omega_{\omega}\left(L_{n}\right)=L_{n}-i \omega J_{n}
$$

on the Virasoro modes.

Given any representation $\mu$ implemented on a space $\mathcal{H}_{\mu}$ via the map $\rho_{\mu}: \hat{H}_{4} \rightarrow$ $\operatorname{End}\left(\mathcal{H}_{\mu}\right)$ one can define a new representation $\mu_{\omega}$ which acts on the same space. The new representation $\mu_{\omega}$ is implemented through the concatenation $\rho_{\mu_{\omega}}=\rho_{\mu} \circ \Omega_{-\omega}$. In view of its construction it is termed spectral flow representation. The previous relations, (A.12) and (A.13), imply a simple formula for the characters. Indeed, it is straightforward to relate the character of $\mu_{\omega}$ to that of the unflowed representation $\mu$. A simple algebraic manipulation within the trace implies the following formula for their characters

$$
\chi_{\mu_{\omega}}^{\hat{H}_{4}}(q, z, u)=u^{-\omega} \chi_{\mu}^{\hat{H}_{4}}\left(q, z q^{-\omega}, u\right) .
$$

In order to simplify the notation we identify the label $\mu_{\omega=0}$ with $\mu$ whenever there is no chance of confusion.

In contrast to the case of affine Lie algebras based on compact real forms of finite dimensional semisimple Lie algebras the spectral flow representations of $\hat{H}_{4}$ are not equivalent to the ordinary highest weight representations introduced before. Instead as can be seen from formula (A.14) they allow to extend the range of the values of $p$ from the interval $(0,1)$ to the full real axis.

There is an alternative expression for the characters of the discrete series which we used repeatedly in our derivation of the coset characters. We can write

$$
\chi_{( \pm, p, j)_{\mp \omega}}^{\hat{H}_{4}}(q, z, u)=u^{ \pm(p+\omega)} \frac{1}{\eta(q)^{4}} \sum_{n \in \mathbb{Z}} z^{j \mp n} \sum_{m=0}^{\infty}(-1)^{m} q^{h_{( \pm, p, j)} \pm \omega(j \mp n)+\frac{m}{2}(m+2 n+1)}
$$

where $\omega \in \mathbb{N}$. With the upper sign this formula is valid for $|q|^{-\omega}<|z|<|q|^{-1-\omega}$ while it is valid for $|q|^{1+\omega}<|z|<|q|^{\omega}$ if we choose the lower sign. This expression for the characters follows from the identity

$$
\frac{1}{(1-z) \prod_{n=1}^{\infty}\left(1-z q^{n}\right)\left(1-z^{-1} q^{n}\right)}=\sum_{n \in \mathbb{Z}} z^{n} \sum_{m=0}^{\infty}(-1)^{m} \frac{q^{\frac{m}{2}(m+2 n+1)+\frac{1}{12}}}{\eta(q)^{2}},
$$


where $|q|<|z|<1$ is assumed. This relation, originally proven in [45], can also be derived in the following way 46]. We first write

$$
\frac{1}{(1-z) \prod_{n=1}^{\infty}\left(1-z q^{n}\right)\left(1-z^{-1} q^{n}\right)}=\sum_{l \in \mathbb{Z}} f_{l}(q) z^{l}
$$

and then multiply both sides with $z^{-n-1}$ and perform an integral along a contour contained in the interior of the unit circle. On the right hand side of A.17) we simply obtain the coefficient $f_{n}(q)$ while on the left hand side we pick up the residues from the poles at $z=q^{m}, m>0$. The result

$$
f_{n}(q)=\sum_{m=1}^{\infty}(-1)^{m-1} \frac{q^{-m n+\frac{1}{2} m(m-1)+\frac{1}{12}}}{\eta(q)^{2}}=\sum_{m=0}^{\infty}(-1)^{m} \frac{q^{\frac{1}{2}(m+1)(m-2 n)+\frac{1}{12}}}{\eta(q)^{2}},
$$

coincides with (A.16) after the shift $m \rightarrow m+2 n$.

\section{References}

[1] G. T. Horowitz and A. R. Steif, Singular string solutions with nonsingular initial data, Phys. Lett. B 258 (1991) 91.

[2] L. Cornalba and M. S. Costa, A new cosmological scenario in string theory, Phys. Rev. D 66, 066001 (2002) [hep-th/0203031];

L. Cornalba, M. S. Costa and C. Kounnas, A resolution of the cosmological singularity with orientifolds, Nucl. Phys. B 637, 378 (2002) [hep-th/0204261].

[3] H. Liu, G. W. Moore and N. Seiberg, Strings in a time-dependent orbifold, JHEP 0206 (2002) 045 [hep-th/0204168];

H. Liu, G. W. Moore and N. Seiberg, Strings in time-dependent orbifolds, JHEP 0210 (2002) 031 [hep-th/0206182].

[4] G. T. Horowitz and J. Polchinski, Instability of spacelike and null orbifold singularities, Phys. Rev. D 66 (2002) 103512 [hep-th/0206228].

[5] B. Pioline and M. Berkooz, Strings in an electric field, and the Milne universe, JCAP 0311, 007 (2003) [hep-th/0307280];

M. Berkooz, B. Pioline and M. Rozali, Closed strings in Misner space, JCAP 0408, 004 (2004) [hep-th/0405126];

M. Berkooz, B. Durin, B. Pioline and D. Reichmann, Closed strings in Misner space: Stringy fuzziness with a twist, JCAP 0410, 002 (2004) [hep-th/0407216].

[6] M. Berkooz, Z. Komargodski, D. Reichmann and V. Shpitalnik, Flow of geometries and instantons on the null orbifold, JHEP 0512 (2005) 018 [hep-th/0507067].

[7] R. Gueven, Plane waves in effective field theories of superstrings, Phys. Lett. B 191 (1987) 275 ;

D. Amati and C. Klimcik, Strings in a shock wave background and generation of curved geometry from flat space string theory, Phys. Lett. B 210, 92 (1988);

G. T. Horowitz and A. R. Steif, Space-time singularities in string theory Phys. Rev. Lett. 64 (1990) 260;

G. T. Horowitz and A. R. Steif, Strings in strong gravitational fields, Phys. Rev. D 42 (1990) 1950; 
A. A. Tseytlin, String vacuum backgrounds with covariantly constant null Killing vector and $2 D$ quantum gravity, Nucl. Phys. B 390, 153 (1993) [hep-th/9209023].

[8] G. Papadopoulos, J. G. Russo and A. A. Tseytlin, Solvable model of strings in a time-dependent plane-wave background, Class. Quant. Grav. 20 (2003) 969 [hep-th/0211289].

[9] C. R. Nappi and E. Witten, A WZW model based on a nonsemisimple group, Phys. Rev. Lett. 71 (1993) 3751.

[10] G. D'Appollonio and E. Kiritsis, String interactions in gravitational wave backgrounds, Nucl. Phys. B 674 (2003) 80 [hep-th/0305081].

[11] G. D'Appollonio and E. Kiritsis, D-branes and BCFT in Hpp-wave backgrounds, Nucl. Phys. B 712 (2005) 433 [hep-th/0410269].

[12] L. J. Dixon, M. E. Peskin and J. D. Lykken, N=2 superconformal symmetry and $S O(2,1)$ current algebra, Nucl. Phys. B 325, 329 (1989).

[13] K. Gawedzki, Noncompact WZW conformal field theories, [hep-th/9110076].

[14] E. Witten, On string theory and black holes, Phys. Rev. D 44 (1991) 314;

R. Dijkgraaf, H. L. Verlinde and E. P. Verlinde, String propagation in a black hole geometry, Nucl. Phys. B 371, 269 (1992).

[15] K. Bardakci, E. Rabinovici and B. Saering, String models with $c<1$ components, Nucl. Phys. B 299 (1988) 151;

K. Gawedzki and A. Kupiainen, $G / H$ conformal field theory from gauged WZW model, Phys. Lett. B 215 (1988) 119;

K. Gawedzki and A. Kupiainen, Coset construction from functional integrals, Nucl. Phys. B 320 (1989) 625;

D. Karabali and H. J. Schnitzer, BRST quantization of the gauged WZW action and coset conformal field theories, Nucl. Phys. B 329, 649 (1990);

E. Witten, On holomorphic factorization of WZW and coset models, Commun. Math. Phys. 144 (1992) 189.

[16] C. R. Nappi and E. Witten, A closed, expanding universe in string theory, Phys. Lett. B 293 (1992) 309 [hep-th/9206078].

[17] J. Teschner, On structure constants and fusion rules in the $S L(2, \mathbb{C}) / S U(2) W Z N W$ model, Nucl. Phys. B 546, 390 (1999) [hep-th/9712256].

[18] J. M. Maldacena and H. Ooguri, Strings in $A d S_{3}$ and $S L(2, \mathbb{R})$ WZW model. I, J. Math. Phys. 42 (2001) 2929 [hep-th/0001053].

[19] J. M. Maldacena and H. Ooguri, Strings in $A d S_{3}$ and the $S L(2, \mathbb{R})$ WZW model. III: Correlation functions, Phys. Rev. D 65 (2002) 106006 [hep-th/0111180].

[20] J. M. Maldacena, H. Ooguri and J. Son, Strings in $A d S_{3}$ and the $S L(2, \mathbb{R}) W Z W$ model. II: Euclidean black hole, J. Math. Phys. 42 (2001) 2961 [hep-th/0005183].

[21] S. Elitzur, A. Giveon, D. Kutasov and E. Rabinovici, From big bang to big crunch and beyond, JHEP 0206 (2002) 017 [hep-th/0204189].

[22] E. Kiritsis and C. Kounnas, String propagation in gravitational wave backgrounds, Phys. Lett. B 320 (1994) 264 [Addendum-ibid. B 325 (1994) 536] [hep-th/9310202].

[23] K. Sfetsos, Gauging a nonsemisimple WZW model Phys. Lett. B 324 (1994) 335 [hep-th/9311010]. 
[24] I. Antoniadis and N. A. Obers, Plane gravitational waves in string theory, Nucl. Phys. B 423, 639 (1994) [hep-th/9403191].

[25] K. Sfetsos and A. A. Tseytlin, Four-dimensional plane wave string solutions with coset CFT description, Nucl. Phys. B 427 (1994) 245 [hep-th/9404063].

[26] G. D'Appollonio and T. Quella, The diagonal cosets of the Heisenberg group, in preparation.

[27] S. Deser, R. Jackiw and G. 't Hooft, Three-dimensional Einstein gravity: Dynamics of flat space, Annals Phys. 152 (1984) 220.

[28] M. A. Melvin, Pure magnetic and electric geons, Phys. Lett. 8, 65 (1964).

[29] M. Bianchi, G. D'Appollonio, E. Kiritsis and O. Zapata, String amplitudes in the Hpp-wave limit of $A d S_{3} \times S^{3}$, JHEP 0404 (2004) 074 [hep-th/0402004].

[30] D. I. Olive, E. Rabinovici and A. Schwimmer, A Class of string backgrounds as a semiclassical limit of WZW models, Phys. Lett. B 321 (1994) 361 [hep-th/9311081].

[31] A. Giveon, O. Pelc and E. Rabinovici, WZNW models and gauged WZNW models based on a family of solvable Lie algebras, Nucl. Phys. B 462 (1996) 53.

[32] E. Kiritsis and B. Pioline, Strings in homogeneous gravitational waves and null holography, JHEP 0208 (2002) 048 [hep-th/0204004].

[33] F. Dowker, J. P. Gauntlett, D. A. Kastor and J. H. Traschen, Pair creation of dilaton black holes, Phys. Rev. D 49, 2909 (1994) [hep-th/9309075].

[34] F. Dowker, J. P. Gauntlett, S. B. Giddings and G. T. Horowitz, On pair creation of extremal black holes and Kaluza-Klein monopoles, Phys. Rev. D 50, 2662 (1994) [hep-th/9312172].

[35] A. A. Tseytlin, Melvin solution in string theory, Phys. Lett. B 346 (1995) 55 [hep-th/9411198].

[36] J. G. Russo and A. A. Tseytlin, Constant magnetic field in closed string theory: An exactly solvable model Nucl. Phys. B 448, 293 (1995) [hep-th/9411099].

[37] E. Dudas and J. Mourad, D-branes in string theory Melvin backgrounds, Nucl. Phys. B 622, 46 (2002) [hep-th/0110186].

[38] D. Kutasov, J. Marklof and G. W. Moore, Melvin models and diophantine approximation, Commun. Math. Phys. 256, 491 (2005) [hep-th/0407150].

[39] I. Bakas and K. Sfetsos, PP-waves and logarithmic conformal field theories, Nucl. Phys. B 639 (2002) 223 [hep-th/0205006].

[40] E. Kiritsis, Exact duality symmetries in CFT and string theory, Nucl. Phys. B 405 (1993) 109 [hep-th/9302033].

[41] A. Giveon and E. Kiritsis, Axial vector duality as a gauge symmetry and topology change in string theory, Nucl. Phys. B 411 (1994) 487 [hep-th/9303016].

[42] M. D. McGuigan, C. R. Nappi and S. A. Yost, Charged black holes in two-dimensional string theory, Nucl. Phys. B 375 (1992) 421 [hep-th/9111038].

[43] J. H. Horne and G. T. Horowitz, Exact black string solutions in three-dimensions, Nucl. Phys. B 368 (1992) 444 [hep-th/9108001].

[44] P. H. Ginsparg and F. Quevedo, Strings on curved space-times: Black holes, torsion, and duality, Nucl. Phys. B 385, 527 (1992) [hep-th/9202092]. 
[45] A. Pakman, BRST quantization of string theory in $A d S_{3}$, JHEP 0306 (2003) 053 [hep-th/0304230].

[46] S. Ribault and V. Schomerus, Branes in the 2D black hole, JHEP 0402, 019 (2004) [hep-th/0310024].

[47] D. Gepner, Yukawa couplings for Calabi-Yau string compactification, Nucl. Phys. B 311 (1988) 191.

[48] A. B. Zamolodchikov and V. A. Fateev, Operator algebra and correlation functions in the two-dimensional Wess-Zumino $S U(2) \times S U(2)$ chiral model, Sov. J. Nucl. Phys. 43 (1986) 657 [Yad. Fiz. 43 (1986) 1031].

[49] A. Adams, J. Polchinski and E. Silverstein, Don't panic! Closed string tachyons in ALE space-times, JHEP 0110 (2001) 029 [hep-th/0108075].

[50] S. Elitzur and G. Sarkissian, D-branes on a gauged WZW model, Nucl. Phys. B 625, 166 (2002) [hep-th/0108142];

S. Fredenhagen and V. Schomerus, D-branes in coset models, JHEP 0202, 005 (2002) [hep-th/0111189];

T. Quella and V. Schomerus, Asymmetric cosets, JHEP 0302 (2003) 030 [hep-th/0212119]. 